\title{
ОЦЕНИВАНИЕ ОПТИМАЛЬНОГО ПАРАМЕТРА РЕГУЛЯРИЗАЦИИ ВОССТАНОВЛЕНИЯ СИГНАЛА
}

\author{
E. D. Prilepsky, J. E. Prilepsky \\ Aston Institute of Photonic Technologies, Aston University, Birmingham, B47ET, UK
}

\begin{abstract}
Аннотация. В данной работе мы исследуем регуляризующие свойства дискретизации в пространстве выходных сигналов для линейных некорректных операторных уравнений с зашумлеными данными. Суть нового метода заключается в том, чтобы выбирать уровень дискретизации, который выступает в качестве параметра регуляризации в этом контексте, по принципу равенства случайной и детерминистической составляющих погрешностей восстановления входного сигнала. Показано, что данный метод, т.е. решение, дискретное по выходному сигналу, устойчив к малым погрешностям в выходном сигнале, и при этом ошибка восстановления входного сигнала однозначно связана (при заданном уровне погрешности измерения выходного сигнала) с шагом дискретизации выходного сигнала, что позволяет корректно и обоснованно выбирать параметр регуляризации для заданного определенного критерия, например - для заданной наперед желаемой погрешности измерения. Конкретные рассчеты и примеры приведены в явном виде для одномерного случая, что не ограничивает общности предлагаемого метода.
\end{abstract}

Ключевые слова: восстановление сигналов и изображений, линейный регуляризирующий алгоритм оценивания оптимального параметра, задачи регуляризации.

\section{1. Введение}

Математической моделью стационарных измерительных систем является интегральное уравнение первого рода с разностным ядром [1,2]:

$\int g(r 1) h(r-r 1) d r 1=f(r)$,

где $h(r-r 1)$ - импульсный отклик системы (функция рассеяния точки). Хорошо известны возникающие принципиальные и практические трудности при анализе уравнения (1). Ядро преобразования (1), $h(r-r 1)$, отражает сглаживающие свойства прибора или метода измерений в целом. Для реальной измерительной системы возникает задача поиска соответствия между выходным сигналом $f(r)$ и искомым входным сигналом $g(r 1)$, что требует решения интегрального уравнения (1). С математической точки зрения необходимо найти решение интегрального уравнения первого рода (1), что является некорректно поставленной задачей [1-3] в смысле определения, что решение такой задачи чрезвычайно чувствительно к малым изменениям данных имерения (т.е. функции $f(r)$ ). Таким образом, особенностью процесса восстановления входного сигнала является некорректность (1), вытекающая из наличия правой части уравнения и чувствительности решения задачи к поведению сигнала $f(r)[1]$. Причина отсутствия непрерывной зависимости решения от правой части уравнения состоит в том, что для всех физически реализуемых систем частотный спектр импульсного отклика убывает в области высоких пространственных частот. Поэтому при обратном преобразовании от выходного сигнала к входному сигналу высокочастотные составляющие даже очень малой амплитуды будут усиливаться, приводя к разрушению информации о входном сигнале. Отметим, что не смотря на то, что интерес к решению некорретных задач начал возникать более 50 лет назад, подобные задачи и методы их решения 
и регуляризации не потеряли свою актуальность и в настоящее время [4], имея важные применения в задачах теплопроводности $[5,6]$, медицине $[7,8]$, и других многочисленных областях. Новые применения включают разработку систем оптоволоконной связи основанных на методах обработки сигнала с помощью обратной задачи рассеяния (нелинейного преобразования Фурье) [9]. В последнее время для решения некорректных задач предлагаются методы машинного обучения и искуственных нейронных сетей [10].

Существует множество методов нахождения приближенного решения уравнения (1), устойчивых к погрешностям при измерении выходного сигнала [1-3]. Большинство этих методов основано на замене оператора задачи (1) приближенным, регуляризированным оператором, спектр которого (собственные значения) не обращается в нуль и не стремится к нулю для высокочастотных составляющих. Очевидно, что приближенное решение, полученное с таким оператором, будет устойчивым. Существенным вопросом этих методов, представляющим априорную трудность, является выбор конкретного вида регуляризатора и численного значения параметра регуляризации [1]. В настоящей работе некорректность исходной задачи (1) преодолевается посредством дискретизации выходного сигнала. Как будет показано в нашей работе, решение, дискретное по выходному сигналу уравнения (1), устойчиво к малым погрешностям в выходном сигнале даже при точном операторе задачи (1). При таком подходе ошибка восстановления входного сигнала оказывается однозначно связанной (при заданном уровне погрешности измерения выходного сигнала) с шагом дискретизации выходного сигнала, и шаг дискретизации играет роль параметра регуляризации, что позволяет обоснованно подойти к выбору этого параметра. Отметим, что дискретизация выходного сигнала нужна в целях выполнения расчетной схемы измерений. С другой стороны, дискретизация выходного сигнала выступает в качестве регуляризации задачи решения уравнения (1) [11-19].

\section{2. Метод решения задачи}

Рассмотрим для простоты одномерный случай [14]. Предположим, что выходной сигнал $f(r)$ измеряется только в конечном числе $N$ точек, так что уравнение (1) заменяется системой линейных уравнений:

$$
f\left(r_{i}\right)=\int h\left(r_{i}-r 1\right) g(r 1) d r 1, \quad i=1 \ldots N .
$$

Обозначим $f\left(r_{i}\right)=f_{i}, h\left(r_{i}-r 1\right)=h_{i}(r 1)$, и будем считать функции $\left\{h_{i}(r 1)\right\}$ линейно независимыми. Тогда система функций $\left\{h_{i}(r 1)\right\}$ образует базис (в общем случае неортогональный). Как будет показано ниже, уравнение (2), в отличие от уравнения (1), имеет устойчивое решение даже при точном операторе $\hat{h}$ из (1). Более того, погрешность восстановления входного сигнала однозначно связана с интервалом дискретизации выходного сигнала, так что погрешность восстановления входного сигнала растет при уменьшении интервала дискретизации. Другими словами, интервал дискретизации играет роль параметра регуляризации, его величина прямо определяет составляющую погрешности восстановления входного сигнала, обусловленную помехами в выходном сигнале. Можно определить шаг дискретизации, например, по критерию минимума суммарной погрешности, либо критерий минимума одной составляющей погрешности при заданном уровне другой составляющей, либо, наконец, информационный критерий [20,21].

Перейдем теперь к решению задачи восстановления входного сигнала и на этой основе определим составляющие компоненты погрешности: детерминированную составляющую и стохастическую составляющую. Подчеркнем, что даже в том случае, когда по каким либо причинам восстановление входного сигнала по дискретизированному выходному сигналу не производится, решение указанной задачи определяет потенциально содержащуюся в дискретном выходном сигнале информацию о входном сигнале в зависимости от интервала дискретизации и, следовательно, позволяет обоснованно определить интервал дискретизации. 
Рассмотрим уравнение (2), связывающее входной сигнал и дискретный сигнал на выходе. Величины $f\left(r_{i}\right)=f_{i}$ можно рассматривать как проекции входного сигнала $g(r 1)$ на подпространство $H$, натянутое на систему функций $\left\{h_{i}(r 1)\right\}$ :

$$
\left(g, h_{n}\right)=f_{n} .
$$

Величина $\left(g, h_{n}\right)=\int h_{n}(r 1) g(r 1) d r 1$ есть скалярное произведение входного сигнала $g(r 1)$ и функции $h_{n}(r 1)$ в подпространстве $H$. Система функций $\left\{h_{n}(r 1)\right\}$ не является в общем случае полной и образует подпространство в пространстве входных сигналов $g(r 1)$. Тогда регуляризированное решение $g_{H}(r 1)$ можно представить в виде:

$$
g_{H}(r 1)=\sum_{n} q_{n} h_{n}(r 1) .
$$

Коэффициенты $\left\{q_{n}\right\}$ не равны одновременно нулю. Подставляя соотношение (4) в (3), получим систему уравнений для определения коэффициентов $\left\{q_{n}\right\}$ :

$$
\sum_{m} K_{n m} q_{m}=f_{n}
$$

где матрица $K_{n m}$ определяется следующим выражением:

$$
K_{n m}=\int h_{n}(r 1) h_{m}(r 1) d r 1=\left(h_{n}, h_{m}\right) .
$$

Детерминант матрицы $K_{n m}$ есть определитель Грама системы функций $\left\{h_{n}(r 1)\right\}$ и для независимых функций не имеет нулевых собственных значений. Поэтому решение системы уравнений (5) для величин $q_{n}$ единственно [22].

Теперь мы рассмотрим случай безграничного интервала и равномерной дискретизации с интервалом $\Delta x$. Случай ограниченных входных и выходных сигналов был рассмотрен в работе [13]. В рассматриваемом здесь случае решение системы уравнений (5), то есть $\left\{q_{n}\right\}$, и, следовательно, регуляризованное решение для входного сигнала $g_{H}(r 1)$ из (4) могут быть найдены в явной форме. Матрица $K_{n m}$ - бесконечномерная Теплицева матрица, то есть ее элементы зависят только от разности индексов:

$$
K_{n m}=\int_{-\infty}^{\infty} h(n \Delta x-y) h(m \Delta x-y) d y=\int_{-\infty}^{\infty} h((n-m) \Delta x+z) h(z) d z=K(n-m) .
$$

Далее решение системы уравнений (5) и регуляризированный входной сигнал (4) могут быть найдены в явной форме с помощью преобразования Фурье. Введем преобразования Фурье дискретного выходного сигнала $\left\{f_{n}\right\}$ и системы коэффициентов $\left\{q_{n}\right\}$ :

$$
F(\omega)=\sum_{n} f_{n} \exp (-j \omega n \Delta x), \quad Q(\omega)=\sum_{n} q_{n} \exp (-j \omega n \Delta x) .
$$

Величины $F(\omega)$ и $Q(\omega)$ периодичны с периодом $2 \pi / \Delta x$, поэтому значения частоты $\omega$ ограничены интервалом: $-\pi / \Delta x \leq \omega \leq \pi / \Delta x$. Обратные преобразования Фурье имеют вид:

$$
f_{n}=\frac{\Delta x}{2 \pi} \int_{-\pi / \Delta x}^{\pi / \Delta x} F(\omega) \exp (j \omega n \Delta x) d \omega, \quad q_{n}=\frac{\Delta x}{2 \pi} \int_{-\pi / \Delta x}^{\pi / \Delta x} Q(\omega) \exp (j \omega n \Delta x) d \omega .
$$

Выполняя преобразование Фурье уравнения (5), приходим к следующему соотношению: $Q(\omega)=F(\omega) / \lambda(\omega)$, где $\lambda(\omega)$ - собственные значения (спектр) матрицы $K_{n m}$ с матричными элементами $K_{n m}=K(n-m)$ :

$$
\lambda(\omega)=\sum_{n=-\infty}^{\infty} K(n) \exp (-j n \omega \Delta x)=K(0)+2 \sum_{n=1}^{\infty} K(n) \cos (n \omega \Delta x) .
$$

Используя соотношения (9) и (4), найдем регуляризованное решение:

$$
g_{H}(r 1)=\frac{\Delta x}{2 \pi} \int_{-\pi / \Delta x}^{\pi / \Delta x} \frac{F(\omega) \psi(r 1 ; \omega)}{\lambda(\omega)} d \omega,
$$


где введена функция $\psi(r 1 ; \omega)$ :

$$
\psi(r 1 ; \omega)=\sum_{n} h(n \Delta x-r 1) \exp (j n \omega \Delta x) .
$$

Система функций $\{\psi(r 1 ; \omega)\}$ образует ортогональный (но ненормированный) базис в пространстве $H$. Действительно, используя соотношение (7) и равенство

$$
\sum_{n=-\infty}^{\infty} \exp [j(\omega 1-\omega) n \Delta x]=\frac{2 \pi}{\Delta x} \delta(\omega 1-\omega), \quad-\pi / \Delta x \leq \omega, \omega 1 \leq \pi / \Delta x,
$$

(в общем случае сумма экспонент в левой части равна периодической $\delta$-функции [23], однако учитывая интервал изменения $\omega$ и $\omega 1$, мы отсавляем лишь одно нулевое слагаемое), получим:

$$
\int_{-\infty}^{\infty} \psi(r 1 ; \omega) \psi(r 1 ; \omega 1) d r 1=\frac{2 \pi}{\Delta x} \lambda(\omega) \delta(\omega 1-\omega), \quad-\pi / \Delta x \leq \omega, \omega^{`} \leq \pi / \Delta x .
$$

Свяжем теперь функции $\psi(r 1 ; \omega)$ и собственные значения $\lambda(\omega)$ с преобразованием Фурье передаточной функции системы. Обозначим через $n=\left[\frac{r 1}{\Delta x}\right]$ - целую часть, а через $\gamma=\left\{\frac{r 1}{\Delta x}\right\}$ дробную часть величины $\frac{r 1}{\Delta x}$, то есть начальную фазу дискретизации, так что $r 1=(n+\gamma) \Delta x$. Сдвинув начало отсчета в формуле (12), получим:

$$
\psi(r 1 ; \omega)=\exp (j n \omega \Delta x) H^{*}(\omega, \gamma) .
$$

Здесь $H(\omega, \gamma)$ - преобразование Фурье передаточной функции:

$$
H(\omega, \gamma)=\sum_{k} h[(k-\gamma) \Delta x] \exp (-j k \omega \Delta x) .
$$

Поскольку $\gamma=\left\{\frac{r 1}{\Delta x}\right\}$ периодично по $r 1$ с периодом $\Delta x$, то и функция $H(\omega, \gamma)$ периодична по величине $\gamma$ с периодом $\Delta x$. Обратное преобразование Фурье дает:

$$
h[(k-\gamma) \Delta x]=\frac{\Delta x}{2 \pi} \int_{-\pi / \Delta x}^{\pi / \Delta x} H(\omega, \gamma) \exp (j k \Delta x) d \omega .
$$

Выразим $\lambda(\omega)$ через частотную характеристику $H(\omega, \gamma)$. Для этого воспользуемся вначале соотношением (7) (заменив $z$ на $-z$ ) и разделим интервал интегрирования на участки $\Delta x$ :

$$
K(n)=\int_{-\infty}^{\infty} h(n \Delta x-z) h(-z) d z=\sum_{k=-\infty}^{\infty} \int_{k \Delta x}^{(k+1) \Delta x} h(n \Delta x-z) h(-z) d z .
$$

В каждом интервале сделаем замену переменных $z=(k+\gamma) \Delta x$, так что предыдущее выражение приводится к виду:

$$
K(n)=\Delta x \sum_{k=-\infty}^{\infty} \int_{0}^{1} h[(n-k-\gamma) \Delta x] h[-(k+\gamma) \Delta x] d \gamma
$$

Теперь подставим в это выражение в соотношение (16), заменив для удобства вычислений $h[-(k+\gamma) \Delta x]$ на комплексно сопряженный (равный ему в силу вещественности) член. Используя соотношение

$$
\sum_{k=-\infty}^{\infty} \exp [j(\omega-\omega 1) k \Delta x]=\frac{2 \pi}{\Delta x} \delta(\omega 1-\omega), \quad\lfloor\omega\rfloor,\lfloor\omega 1\rfloor \leq \frac{\pi}{\Delta x},
$$

получим:

$$
K(n)=\Delta \mathrm{x}\left(\frac{\Delta x}{2 \pi}\right) \int_{0}^{1} d \gamma \int_{-\pi / \Delta x}^{\pi / \Delta x}[H(\omega, \gamma)\rfloor^{2} \exp (j n \omega \Delta x) d \omega .
$$

Подставив на последнем этапе это выражение в формулу (10), найдем окончательно: 


$$
\lambda(\omega)=\Delta x \int_{0}^{1}\lfloor H(\omega, \gamma)\rfloor^{2} d \gamma .
$$

Физический смысл формулы (17) можно объяснить достаточно просто. Спектр оператора (матрицы $K_{n m}$ ) получается усреднением квадрата модуля частотной характеристики по начальной фазе дискретной выборки выходного сигнала. Одним из следствий такого усреднения является то, что даже если частотная характеристика $H(\omega, \gamma)$ имеет изолированные нули (что имеет место, например, для частотной характеристики текущего среднего), то в спектре $\lambda(\omega)$ эти нули устраняются, так что решение и в таком случае остается регулярным. Подставив в формулу (11) значения $\psi(r 1 ; \omega)(14)$ и $\lambda(\omega)(17)$, окончательно получим регулярное решение в виде:

$$
g_{H}(r 1)=\frac{1}{2 \pi} \int_{-\pi / \Delta x}^{\pi / \Delta x} \frac{F(\omega) \exp (j n \omega \Delta x) H^{*}(\omega, \gamma)}{\int_{0}^{1}\lfloor H(\omega, \gamma)\rfloor^{2} d \gamma} d \omega .
$$

Здесь: $n=[r 1 / \Delta x], \quad \gamma=\{r 1 / \Delta x\}$.

Соотношение (18) позволяет установить вид решения не только в точках дискретизации $r 1=n \Delta x$, но и в промежуточных точках, определяя зависимость от выходного сигнала $g(r 1)$. Для этого заменим $F(\omega)$ соотношением (8), и воспользуемся формулами (2) и (14). В результаты получаем выражение для $g_{H}(r 1)$ в следующем виде:

$$
g_{H}\left(r 1^{\prime}\right)=\int L\left(r 1, r 1^{\prime}\right) g(r 1) d r 1,
$$

где

$$
L\left(r 1, r 1^{\prime}\right)=\frac{1}{2 \pi} \int_{-\pi / \Delta x}^{\pi / \Delta x} \frac{H(\omega, \gamma) H^{*}(\omega, \gamma) \exp \left(j\left(n^{\prime}-n\right) \omega \Delta x\right)}{\int_{0}^{1}\left[\left.H(\omega, \gamma)\right|^{2} d \gamma\right.} d \omega .
$$

В данном выражении: $n=[r 1 / \Delta x], n^{\prime}=\left[r 1^{\prime} / \Delta x\right], \gamma=\{r 1 / \Delta x\}, \gamma^{\prime}=\left\{r 1^{\prime} / \Delta x\right\}$. Оператор $\hat{L}$ есть оператор проектирования на подпространство $H$. Можно легко убедиться в том, что $\widehat{L}^{2}=$ $\hat{L}$. Чтобы представить себе вид регуляризированного сигнала $g_{H}(r 1)$ в зависимости от исходного входного сигнала $g(r 1)$, рассмотрим вначале случай, когда передаточная функция $h(r 1)$ мало меняется в пределах интервала дискретизации $\Delta x$. В этом случае частотная характеристика $H(\omega, \gamma)$ практически не зависит от начальной фазы дискретизации $\gamma$, и поэтому из (22) получаем:

$$
L\left(r 1, r 1^{\prime}\right) \cong \frac{1}{2 \pi} \int_{-\pi / \Delta x}^{\pi / \Delta x} \exp \left[j\left(n^{\prime}-n\right) \omega \Delta x\right] d \omega=\frac{1}{\Delta x} \delta_{n^{\prime}, n}=\frac{1}{\Delta x}\left\{\begin{array}{l}
1, n^{\prime}=n \\
0, n^{\prime} \neq n .
\end{array}\right.
$$

Окончательно, функция $g_{H}(r 1)$ на интервале $n \Delta x<r 1<(n+1) \Delta x$ будет равна:

$$
g_{H}(r 1) \cong \frac{1}{\Delta x} \int_{n \Delta x}^{(n+1) \Delta x} g\left(r 1^{\prime}\right) d r 1^{\prime} ; \quad n \Delta x<r 1<(n+1) \Delta x .
$$

Таким образом, восстановленный входной сигнал $g_{H}(r 1)$ есть ступенчатая аппроксимация исходного входного сигнала $g(r 1)$, причем значение $g_{H}(r 1)$ на каждом интервале $\Delta x$ есть среднее значение исходного входного сигнала $g(r 1)$ на этом интервале. В общем случае, когда передаточная функция $h(r 1)$ заметно меняется в пределах интервала дискретизации $\Delta x$, то значения восстановленного сигнала $g_{H}(r 1)$ определяются взвешенным средним исходного входного сигнала $g(r 1)$ не только по данному интервалу, но и по соседним интервалам. Вид взвешивающей функции определяется передаточной функцией $h(r 1)$.

\section{3. Погрешность восстановления входного сигнала}

Полученное решение задачи позволяет определить одну из составляющих погрешности детерминированную составляющую $\varepsilon_{1}$ : 


$$
\varepsilon_{1}^{2}=\frac{\left\|g-g_{H}\right\|^{2}}{\|g\|^{2}} .
$$

Если частота дискретизации достаточно велика, так что передаточная функция $h(r 1)$ мало меняется на интервале дискретизации $\Delta x$, то $g_{H}(r 1)$ определяется формулой (24), и детерминированная составляющая погрешности $\varepsilon_{1}$ есть попросту погрешность ступенчатой аппроксимации. Если в пределах интервала $\Delta x$ входной сигнал меняется плавно, без резких всплесков и скачков, то его можно разложить на интервалах $\Delta x$ в ряд Тейлора и ограничиться линейным членом: $g_{H}(r 1) \cong g(n \Delta x)+g^{\prime}(n \Delta x)(r 1-n \Delta x) ; n \Delta x<r 1<(n+1) \Delta x$. Значение $g_{H}(r 1)$ на каждом интервале будет равно, в соответствии с формулой (24): $g_{H}(r 1) \cong g(n \Delta x)+g^{\prime}(n \Delta x) \frac{\Delta x}{2}$. Полученные соотношения позволяют выразить детерминированную составляющую погрешности $\varepsilon_{1}$ через величину интервала дискретизации $\Delta x$. Вычислим вначале величину

$$
\begin{aligned}
\left\|g-g_{H}\right\|^{2} & =\sum_{n} \int_{n \Delta x}^{(n+1) \Delta x}\left(g(r 1)-g_{H}(r 1)\right)^{2} d r 1=\frac{1}{12}(\Delta x)^{2} \sum_{n}\left(g^{\prime}(n \Delta x)\right)^{2} \Delta x \cong \\
& \cong \frac{1}{12} \Delta x^{2} \int g^{\prime}(r 1)^{2} d r 1 .
\end{aligned}
$$

Из занного выражения, для детерминированной составляющей погрешности $\varepsilon_{1}$ находим:

$$
\varepsilon_{1} \cong \frac{\Delta x}{\Theta}
$$

где параметр $\Theta$ равен:

$$
\Theta=\left(\int\left(g^{\prime}(r 1)\right)^{2} \mathrm{~d} r 1 / 12 \int(g(r 1))^{2} \mathrm{~d} r 1\right)^{-1 / 2} .
$$

Величина $\Theta$, определяемая (27), в рассматриваемом приближении не зависит от интервала дискретизации $\Delta x$ и является параметром, характеризующим свойства входного сигнала: чем меньше параметр $\Theta$, тем быстрее и резче меняется входной сигнал. Формула (26) получена разложением в ряд Тейлора с учетом первого члена разложения. Поэтому она тем точнее, чем меньше величина погрешности $\varepsilon_{1}$.

Рассмотрим случайную составляющую погрешности восстановления входного сигнала. В реальных условиях на выходной дискретный сигнал накладываются погрешности и помехи, например, шум квантования. Эти шумы делают невозможным точное восстановление даже регуляризированного решения $g_{H}(r 1)$. Обозначим через $n_{l}$ случайную погрешность в дискретном выходном сигнале. Тогда, в соответствии с формулой $(8), \Delta F(\omega)=$ $\sum_{l} n_{l} \exp (-j \omega l \Delta x)$ будет случайной погрешностью спектра выходного сигнала. Случайная составляющая погрешности регулярного решения задачи $\Delta g_{H}$, как вытекает из (11), будет равна: $\Delta g_{H}(r 1)=\frac{\Delta x}{2 \pi} \int_{-\pi / \Delta x}^{\pi / \Delta x} \frac{\Delta F(\omega) \psi(r 1 ; \omega)}{\lambda(\omega)} d \omega$. Пользуясь ортогональностью функций $\psi(r 1 ; \omega)$ из (13), найдем относительную случайную составляющую погрешности восстановления входного сигнала $\varepsilon_{2}^{2}$, то есть отношение мощности помехи к мощности сигнала, $\left\|\Delta g_{H}(r 1)\right\|^{2} /$ $\left\|g_{H}(r 1)\right\|^{2}$ :

$$
\varepsilon_{2}^{2}=\int_{-\pi / \Delta x}^{\pi / \Delta x} \frac{G(\omega)}{\lambda(\omega)} d \omega / \int_{-\pi / \Delta x}^{\pi / \Delta x} \frac{G_{0}(\omega)}{\lambda(\omega)} d \omega
$$

где $G(\omega)$ - спектральная плотность мощности помехи в выходном сигнале, а $G_{0}(\omega)$ спектральная плотность выходного сигнала. В наиболее неблагоприятном случае, когда спектр помехи сосредоточен вблизи наименьшего значения $\lambda(\omega)$, равного $\lambda_{\min }$, а спектр сигнала сосредоточен вблизи наибольшего значения $\lambda(\omega)$, равного $\lambda_{\max }$, из формулы (28) получаем (Задача А): 


$$
\varepsilon_{2}^{\prime}=\sqrt{\frac{\lambda_{\max }}{\lambda_{\min }} \sqrt{\frac{P}{P_{0}}}}
$$

где $P=\int_{-\pi / \Delta X}^{\pi / \Delta X} G(\omega) d \omega-$ мощность помехи, а $P_{0}=\int_{-\pi / \Delta x}^{\pi / \Delta x} G_{0}(\omega) d \omega-$ мощность сигнала. Если помеха равномерно распределена по спектру в пределах диапазона $(-\pi / \Delta x, \pi / \Delta x)$, получается меньшее значение погрешности (Задача В):

$$
\varepsilon_{2}^{\prime \prime}=\sqrt{\lambda_{\max }\left(\frac{1}{\lambda}\right)_{\mathrm{av}}} \sqrt{\frac{P}{P_{0}}}
$$

где $\left(\frac{1}{\lambda}\right)_{\mathrm{av}}=\frac{\Delta x}{2 \pi} \int_{-\pi / \Delta x}^{\pi / \Delta x} \frac{d \omega}{\lambda(\omega)}$. Величина $P_{0} / P-$ есть отношение сигнал/шум на выходе, для выходного сигнала, а величина $\varepsilon_{2}^{-2}$ - отношение сигнал/шум на входе, для входного сигнала. Поэтому коэффициент генерации шума $k$, равный отношению сигнал/шум на входе деленному на отношение сигнал/шум на выходе дается, в наиболее неблагоприятном случае, как вытекает из (29), следующим выражением:

$$
k^{\prime}=\lambda_{\max } / \lambda_{\min },
$$

а при равномерном распределении помехи по спектру, как следует из (30):

$$
k^{\prime \prime}=\lambda_{\max }\left(\frac{1}{\lambda}\right)_{\mathrm{av}}
$$

Погрешности $\varepsilon_{2}^{\prime}$ и $\varepsilon_{2}^{\prime \prime}$ связаны с коэффициентами генерации шума $k^{\prime}$ и $k^{\prime \prime}$ соотношениями:

$$
\varepsilon_{2}^{\prime}=\sqrt{k^{\prime}} \sigma, \quad \varepsilon_{2}^{\prime \prime}=\sqrt{k^{\prime \prime}} \sigma,
$$

где $\sigma^{2}=P / P_{0}$ - относительная мощность помехи, то есть отношение шум/сигнал на выходе. Поскольку величины $\lambda_{\max }, \lambda_{\min },(1 / \lambda)_{\mathrm{av}}$ определяются видом передаточной функции и величиной интервала дискретизации, то полученные соотношения (28)-(32) и соотношения для детерминированной составляющей погрешности $\varepsilon_{1},(25)$ и (26), позволяют обоснованно подойти к определению оптимального интервала дискретизации. Поскольку величина $\varepsilon_{1} \mathrm{c}$ ростом $\Delta x$ увеличивается, а величина $\varepsilon_{2}$ - уменьшается, то такой оптимум существует. В простейшем случае оптимальное значение интервала дискретизации $\Delta x$ можно найти из условия $\varepsilon_{1}=\varepsilon_{2}$ или из условия минимума суммы составляющих погрешностей $\varepsilon_{1}+\varepsilon_{2}$.

\section{4. Пример расчета}

В качестве примера рассмотрим систему в виде простого апериодического звена с передаточной функцией:

$$
h(r 1)= \begin{cases}\alpha \exp (-\alpha r 1), & r 1>0, \\ 0, & r 1<0 .\end{cases}
$$

Величина $\alpha^{-1}$ - постоянная времени звена. Вычислим величину $H(\omega ; \gamma)(15)$. Подставляя в $(15)$ формулу (34), получим:

$$
H(\omega ; \gamma)=\alpha \sum_{k=1}^{\infty} \exp [-((k-\gamma) \Delta x-j k \omega \Delta x)]=H(\omega) \exp (\alpha \gamma \Delta x)
$$

где $H(\omega)=\alpha \sum_{k=1}^{\infty} \exp [-(\alpha+j \omega) \Delta x k]=\alpha(\exp [(\alpha+j \omega) \Delta x]-1)^{-1} . \quad$ Величина $H(\omega) \quad$ не зависит от дробной части $\gamma$, поэтому: 
$\int_{0}^{1}|H(\omega ; \gamma)|^{2} d \gamma=q(\alpha)|H(\omega)|^{2}$, где $q(\alpha)=\int_{0}^{1} \exp (2 \alpha \gamma \Delta x) d \gamma=(\exp (2 \alpha \Delta x)-1) /(2 \alpha \Delta x)$.

Из формулы (18) для $g_{H}(r 1)$ будем иметь:

$$
g_{H}(r 1)=\frac{\exp (\alpha \gamma \Delta x)}{2 \pi \alpha q(\alpha)} \int_{-\pi / \Delta x}^{\pi / \Delta x} F(\omega) \exp (j n \omega \Delta x)[\exp [(\alpha+j \omega) \Delta x]-1] d \omega .
$$

Подставляя сюда формулу (8), окончательно получим регулярное решение:

$$
g_{H}(r 1)=\frac{2 \exp (\alpha \gamma \Delta x)}{\exp (2 \alpha \Delta x)-1}\left[f_{n+1} \exp (\alpha \Delta x)-f_{n}\right]
$$

где $n=[r 1 / \Delta x] ; \gamma=\{r 1 / \Delta x\}$. Решение есть ступенчатая кривая, модулированная множителем $\exp [\alpha\{r 1 / \Delta x\} \Delta x]$. Если $\alpha \Delta x<<1$, то модуляция исчезает и решение приближается к ступенчатой кривой:

$$
g_{H}(r 1) \cong \frac{1}{2 \alpha \Delta x}\left[f_{n+1}-f_{n}\right]
$$

Величину $\lambda(\omega)$ находим из выражения (17):

$$
\lambda(\omega)=q(\alpha) \Delta x|H(\omega)|^{2}=\alpha \Delta x q(\alpha)[\exp (2 \alpha \Delta x)+1-2 \exp (\alpha \Delta x) \cos (\omega \Delta x)]^{-1} .
$$

В результате мы получаем следующие соотнонения:

$$
\begin{aligned}
\lambda_{\max } & =\alpha^{2} \Delta x q(\alpha)(\exp (\alpha \Delta x)-1)^{-2}, \quad \lambda_{\min }=\alpha^{2} \Delta x q(\alpha)(\exp (\alpha \Delta x)+1)^{-2}, \\
\left(\frac{1}{\lambda}\right)_{\mathrm{av}} & =\frac{1}{\alpha^{2} \Delta x q(\alpha)}(\exp (2 \alpha \Delta x)+1) .
\end{aligned}
$$

Коэффициенты генерации шума (31) и (32) будут равны

$$
k^{\prime}=\frac{(\exp (\alpha \Delta x)+1)^{2}}{(\exp (\alpha \Delta x)-1)^{2}}, \quad k^{\prime \prime}=\frac{(\exp (2 \alpha \Delta x)+1)}{(\exp (\alpha \Delta x)-1)^{2}} .
$$

Определение оптимального интервала дискретизации из простейшего уравнения $\varepsilon_{1}=\varepsilon_{2}$ при наиболее неблагоприятной помехе приводит к уравнению (Задача А):

$$
\Delta x=\frac{(\exp (\alpha \Delta x)+1)}{(\exp (\alpha \Delta x)-1)} \sigma \Theta .
$$

Аналогично, при равномерном распределении помехи по спектру для оптимального интервала дискретизации получается уравнение (Задача В):

$$
\Delta x=\frac{\sqrt{\exp (2 \alpha \Delta x)+1}}{(\exp (\alpha \Delta x)-1)} \sigma \Theta
$$

На рис. 1 показаны графики зависимости решений $y_{\mathrm{opt}}=\alpha \Delta x_{\mathrm{opt}}$ от величины параметра $b=\alpha \Theta \sigma$. Решение в предельных случаях легко находим аналитически. При $\alpha \Delta x \ll 1$ решение уравнения (36) будет: $\Delta x_{\mathrm{opt}} \cong \sqrt{2 \sigma \Theta / \alpha}$, а решение уравнения (37) будет: $\Delta x_{\mathrm{opt}} \cong \sqrt{\sqrt{2 \sigma \Theta} / \alpha}$; $\sigma \Theta \alpha \ll 1$. Величины составляющих погрешности будут равны: $\varepsilon_{1}=\varepsilon_{2}^{\prime}=\sqrt{2 \sigma / \alpha \Theta}=$ $2 \sigma / \alpha \Delta x_{\text {opt }}$. Поскольку $\alpha \Delta x_{\text {opt }} \ll 1$, то при восстановлении происходит усиление помехи и восстановление оказывается возможным только при достаточно малой величине помехи $\sigma$ в выходном сигнале. (В оптике и радиодиапазоне этому случаю соответствует так называемое сверхразрешение [24], поскольку входной сигнал восстанавливается на интервале $\Delta x$ значительно меньшем интервала усреднения $\alpha^{-1}$ передаточной функции). В задачах обработки изображений в оптическом и радиодиапазоне такая ситуация хорошо известна. При 
$\alpha \Delta x \gg 1$ как для уравнения (36), так и для уравнения (37): $\Delta x_{\mathrm{opt}} \cong \sigma \Theta ; \sigma \Theta \alpha \gg 1$. В этом случае $\varepsilon_{1}=\varepsilon_{2}^{\prime}=\sigma$, и усиление шума при восстановлении входного сигнала не происходит. Легко также установить зависимость случайной составляющей погрешности $\varepsilon_{2}$ от величины детерминированной составляющей погрешности $\varepsilon_{1}$ :

$$
\varepsilon_{2}^{\prime}=\sigma \frac{\exp \left(\alpha \Theta \varepsilon_{1}\right)+1}{\exp \left(\alpha \Theta \varepsilon_{1}\right)-1} ; \quad \varepsilon_{2}^{\prime \prime}=\sigma \frac{\sqrt{\exp \left(2 \alpha \Theta \varepsilon_{1}\right)+1}}{\exp \left(\alpha \Theta \varepsilon_{1}\right)-1} .
$$

Из формулы (39) ясно, что стремление уменьшить одну из составляющих погрешности приводит к увеличению другой составляющей. Так, в области сверхразрешения $\alpha \Theta \varepsilon_{1}<<1$ имеем: $\varepsilon_{2}^{\prime} \cong 2 \sigma / \alpha \Theta \varepsilon_{1} ; \varepsilon_{2}^{\prime \prime} \cong \sqrt{2 \sigma} / \alpha \Theta \varepsilon_{1}$.

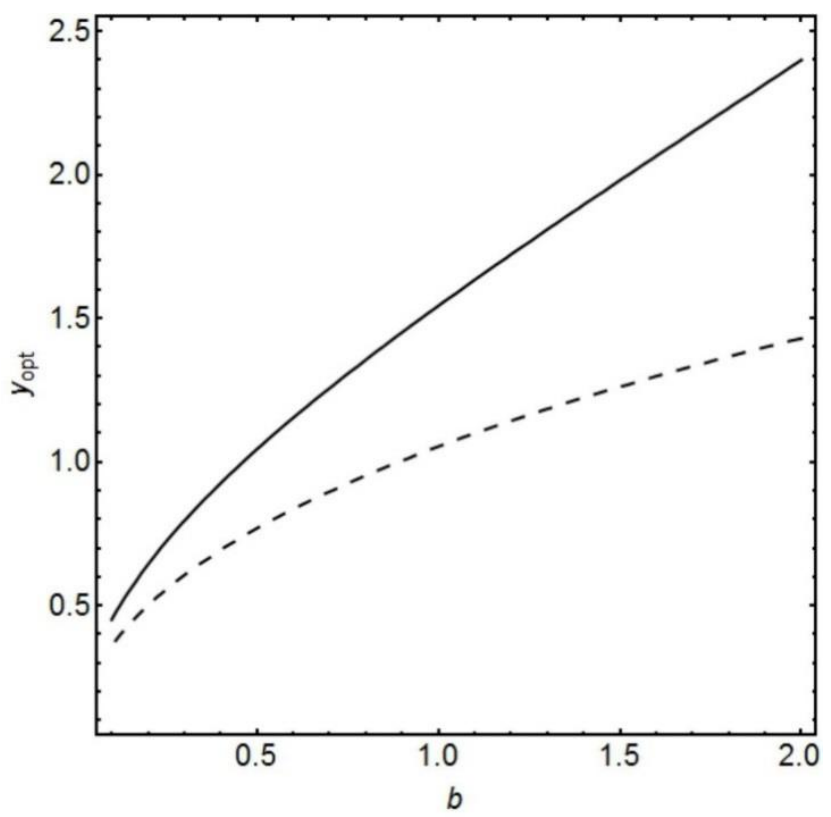

Рис. 1. Зависимости оптимальных решений $y_{\mathrm{opt}}=\alpha \Delta x_{\mathrm{opt}}$ от величины параметра $b=\alpha \Theta \sigma$. Сплошная кривая отвечает случаю Задачи А, пунктирная кривая соответствует случаю Задачи В. Из приведенного рисунка видно, что при увеличении величины параметра $b$ увеличивается оптимальный шаг дискретизации выходного сигнала. При уменьшении параметра b шаг дискретизации выходного сигнала для задач А и В практически совпадает.

Формула (39) дает решение задачи о нахождении оптимального интервала дискретизации в другой постановке: минимальное значение случайной составляющей погрешности $\varepsilon_{2}^{\prime}$ при заданной детерминированной составляющей погрешности $\varepsilon_{1}$. Действительно, уменьшение интервала $\Delta x$ приводит к увеличению случайной составляющей погрешности $\varepsilon_{2}$. Таким образом появляется возможность регулировать величину случайной составляющей погрешности при восстановлении входного сигнала путем выбора шага дисретизации при заданной детерминестической составляющей погрешности. На рис. 2 показаны зависимости величины случайной составляющей погрешности $\varepsilon_{2}$ от детерминированной составляющей погрешности $\varepsilon_{1}$.

\section{5. Обсуждение результатов}

Интересно сопоставить полученное регуляризированное решение с известными регуляризированными решениями. Так, в методе А.Н. Тихонова [1] регуляризированное решение $g_{R}(r 1)$ получается в виде:

$$
g_{R}(r 1)=\frac{1}{2 \pi} \int_{-\infty}^{\infty} \frac{F(\omega) \exp (j \omega r 1) H^{*}(\omega)}{|H(\omega)|^{2}+\alpha^{2}} d \omega,
$$


где $H(\omega)$ - преобразование Фурье передаточной функции, $\alpha$ - параметр регуляризации, демпфирующий малые значения частотной характеристики $H(\omega)$ в области высоких частот. Регуляризирующим параметром в соотношении (18) является интервал дискретизации $\Delta x$. Демпфирование малых значений частотной характеристики в области высоких частот достигается за счет сворачивания спектра в интервале периодичности $-\pi / \Delta x<\omega<\pi / \Delta x$, a устранение изолированных нулей частотной характеристики - за счет усреднения частотной характеристики по интервалу дискретизации $\Delta x$. Сравним решение $g_{H}(r 1)(18)$ с тем, которое получается при замене интеграла в исходном соотношении (1) на сумму, то есть когда дискретизации подвергается не только выходной сигнал, но также входной сигнал и передаточная функция. При этом уравнение (1) заменяется на систему линейных уравнений: $\sum_{m} h((n-m) \Delta x) g_{m}=f_{n}$, где $g_{m}=g(m \Delta x)$ - дискретный входной сигнал. Решение этой системы уравнений имеет вид:

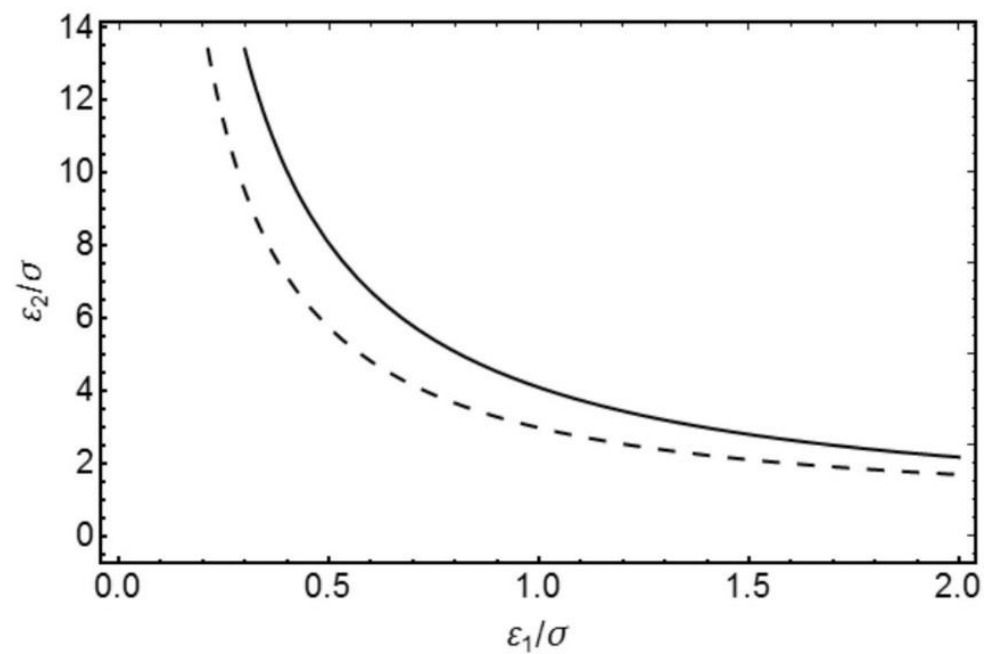

Рис. 2. Зависимости величиньл случайной составляющей погрешности $\varepsilon_{2}$ от детерминированной составляющей погрешности $\varepsilon_{1}$, рассчитаные при величине параметра $b=\alpha \Theta \sigma=0.5$. Сплошная линия представляет зависимость $\varepsilon_{2}^{\prime}$ от $\varepsilon_{1}$ (Задача A), а пунктирная линия - это зависимость $\varepsilon_{2}^{\prime \prime}$ от $\varepsilon_{1}$ (Задача В). Из рисунка ясно, что стремление уменьшить одну из составляющих погрешности приводит к увеличению другой составляющей.

$$
g(n \Delta x)=g_{n}=\frac{1}{2 \pi} \int_{-\pi / \Delta x}^{\pi / \Delta x} \frac{F(\omega) \exp (j n \omega \Delta x)}{H(\omega)} d \omega
$$

где $H(\omega)=H(\omega, 0)$. В тех же дискретных точках $r 1=n \Delta x$ регулярное решение (18) дает:

$$
g_{H}(n \Delta x)=\frac{1}{2 \pi} \int_{-\pi / \Delta x}^{\pi / \Delta x} \frac{F(\omega) \exp (j n \omega \Delta x) H^{*}(\omega ; \gamma)}{\int_{0}^{1}|H(\omega ; \gamma)|^{2} d \gamma} d \omega .
$$

Уравнения (40) и (18) показывают, что в решении $g_{H}(n \Delta x)$ по сравнению $c g(n \Delta x)$ устранены изолированные нули частотной характеристики. Поэтому, например, если в помехе имеются составляющие, частота которых попадает на изолированные нули функции $H(\omega)$, то решение (40) будет неустойчивым, в то время как решение (18) остается устойчивым, то есть резкого нарастания помехи в решении $g_{H}(n \Delta x)$ не происходит. Отметим, что при полной дискретизации (40) шаг дискретизации не связывается с видом ядра уравнения, и решение (40) зависит от начальной фазы дискретизации. Иными словами, в решении (40) не используется вся информация, содержащаяся в промежуточных (между отсчетами) значениях ядра.

\section{6. Заключение}

В предлагаемом методе регулярного восстановления входного сигнала естественным параметром регуляризации является шаг дискретизации выходного сигнала. Поэтому возникает задача о выборе шага дискретизации. Подчеркнем, что по дискретным отсчетам 
выхода нужно восстановить не выходной сигнал, а входной сигнал. Поэтому теорема Котельникова [1] в данной постановке неприменима: например, по теореме Котельникова частота дискретизации должна превышать частоту среза частотной характеристики вдвое, но если взять ядра с крутым частотным срезом и частоту дискретизации больше двойной частоты среза, то вклад помехи неограничен. Погрешность восстановления состоит из двух составляющих: погрешность от замены непрерывного выходного сигнала с бесконечным числом степеней свободы его дискретным аналогом с конечным числом степеней свободы (детерминированная составляющая погрешности), и погрешность, обусловленную шумом в выходном сигнале (случайная составляющая погрешности). C уменьшением шага дискретизации детерминистическая погрешность уменьшается, но случайная составляющая погрешности растет. Таким образом, компромисс между этими составляющими (минимум суммарной погрешности) определяет шаг дискретизации в задаче оптимизации. Если интервал корреляции помехи меньше шага дискретизации, то возможна фильтрация помехи. Отметим, что такая фильтрация (наложение совокупности изображений, полученных от сдвинутых гребенок) не повышает ранг матрицы $K_{n m}$, то есть несущественно усложняет вычисления. Поскольку при фильтрации уменьшается случайная составляющая погрешности, то это позволяет уменьшить шаг дискретизации $\Delta x$, то есть снизить и детерминированную составляющую. При этом возникает задача оптимизации, которую, окончательно, можно сформулировать так: определение новой величины шага регуляризации и, таким образом, получение новой величины параметра регуляризации и соответсвующего ему улучшенного рещения задачи восстановления входного сигнала.

Авторы благодарны UK EPSRC Programme Grant UNLOC EP/J017582/1 за поддержку данных исследований.

\section{СПИСОК ЛИТЕРАТУРЫ}

1. А. Н. Тихонов, В. Я. Арсенин, Методы решения некорректных задач (М.: Наука, 1979).

2. В. А. Морозов, Методы регуляризации неустойчивых задач (М.: Изд-во Московс. ун-та, 1987).

3. А. Б. Бакушинский, А. В. Гончарский, Некорректные задачи. Численные методы и приложения (М.: Изд-во Московс. ун-та, 1989).

4. M. Benning and M. Burger, (2018). Modern regularization methods for inverse problems. Acta Numerica, 27, 1-111 (2018). DOI:10.1017/S0962492918000016

5. V. P. Tanana and A. I. Sidikova, Optimal Methods for Ill-Posed Problems. With Applications to Heat Conduction. (Berlin, Boston: De Gruyter, 2018).

6. K. Mulani, P. Talukdar, A. Das, and R. Alagirusamy, Performance analysis and feasibility study of ant colony optimization, particle swarm optimization and cuckoo search algorithms for inverse heat transfer problems. International Journal of Heat and Mass Transfer, 89, 359-378 (2015). DOI: 10.1016/j.ijheatmasstransfer.2015.05.015

7. M. Stille, M. Kleine, J. Hägele, J. Barkhausen, and T. M. Buzug, Augmented likelihood image reconstruction. IEEE Transactions on Medical Imaging, 35,158-173 (2016). DOI:

10.1109/TMI.2015.2459764

8. T. Gass, G. Székely, and O. Goksel, Consistency-based rectification of nonrigid registrations. Journal of Medical Imaging, 2(1), 014005 (2015). DOI: 10.1117/1.JMI.2.1.014005 
9. S. K. Turitsyn, J. E. Prilepsky, S. T. Le, S. Wahls, L. L. Frumin, M. Kamalian, and S. A. Derevyanko, Nonlinear Fourier transform for optical data processing and transmission: advances and perspectives, Optica 4, 307-322 (2017). DOI: 10.1364/OPTICA.4.000307

10. J. Adler and O. Öktem, (2017). Solving ill-posed inverse problems using iterative deep neural networks. Inverse Problems, 33, 124007 (2017). DOI: 10.1088/1361-6420/aa9581

11. B. Kaltenbacher, Regularization by projection with a posteriori discretization level choice for linear and nonlinear ill-posed problems, Inverse Problems, 16, 1523-1539 (2000). DOI: $10.1088 / 0266-5611 / 16 / 5 / 322$

12. B. Kaltenbacher and J. Offtermatt, A convergence analysis of regularization by discretization in preimage space, Mathematics of Computation, 81, 2049-2069 (2012). DOI: 10.1090/S0025-57182012-02596-8

13. B. Kaltenbacher (Blaschke), H. W. Engl, W. Grever, and M. Klibanov, An application of Tikhonov regularization to phase retrieval, Nonlinear World, 3, 771-786 (1996).

14. B. Kaltenbacher, Boundary observability and stabilization for westervelt type wave equations without interior damping, Applied Mathematics and Optimization, 62, 381-410 (2010). DOI: 10.1007/s00245-010-9108-7

15. D. V. Dovnar and K. G. Predko, Method of eliminating rectilinear uniform blurring of an image, Optoelectronics, Instrumentation and Data Processing (English translation of Avtometriya), Issue 6, 100-103 (1984).

16. D. V. Dovnar and K. G. Predko, Use of orthogonalization of the mappings of basis functions for regularized restoration of a signal, USSR Computational Mathematics and Mathematical Physics, 26, 13-21 (1986). DOI: 10.1016/0041-5553(86)90070-4

17. Yu. E. Voskoboynikov, Estimation of the optimal regularization parameter of an iterative wavelet algorithm for signal recovery, Optoelectronics, Instrumentation and Data Processing, 49, 115-123 (2013). DOI: 10.3103/S875669901

18. Ю. Е. Воскобойников, В. А. Литасов, Устойчивый алгоритм восстановления изображения при неточно заданной аппаратной функции, Автометрия, 42, №6, 3-15 (2006).

19. S. Pereverzev and E. Schock, On the adaptive selection of the parameter in regularization of illposed problems, SIAM J. Numer. Anal., 43, 2060-2076 (2005). DOI:

$10.1137 /$ S00361429034338419

20. M. Y. Mints and E. D. Prilepskii, Image discretization method applied for extended object restoration, Optika I Spectroskopiya, 75, 696-701 (1993).

21. S. P. Luttrell, A new method of sample optimization, Optica Acta, 32(3), 255-257 (1985). DOI: $10.1080 / 713821739$

22. B. R. Frieden, Image-restoration using a norm of maximum information, Optical Engineering, 19, 290-296 (1980). DOI: $10.1117 / 12.7972512$

23. K. Kido, in Digital Fourier Analysis: Fundamentals. Undergraduate Lecture Notes in Physics. Chapter 4: Discrete Fourier Transform. (New York: Springer, 2015). DOI: 10.1007/978-1-46149260-3_4

24. М. Борн, Э. Вольф, Основы оптики (М.: Наука, 1973). 